\title{
A Context-aware and Intelligent Framework for the Secure Mission Critical Systems
}

\author{
${ }^{1}$ Usman Sikandar, ${ }^{1}$ Muhammad Taha, ${ }^{2}$ Sohail Sarwar, ${ }^{3} \mathrm{M}$ Safyan, ${ }^{4}$ Zia Ul Qayyum, \\ ${ }^{5}$ Amjad Ali, ${ }^{6}$ Muddesar Iqbal \\ ${ }^{1}$ Karachi Institute of Economics and Technology, Karachi, Pakistan. \\ ${ }^{2}$ National University of Science and Technology, Islamabad, Pakistan. \\ ${ }^{3}$ LSBU England, ${ }^{3}$ Govt. College University Lahore, Pakistan, \\ ${ }^{4}$ Allama Iqbal Open University, Islamabad, Pakistan \\ ${ }^{5}$ COMSATS Institute of Technology, Islamabad, Lahore Campus, Pakistan \\ ${ }^{6}$ School of Engineering, London South Bank University, UK \\ Corresponding Author: Muddesar Iqbal (m.iqbal@1sbu.ac.uk)
}

\begin{abstract}
Recent technological advancements in pervasive systems have shown the potential to address challenges in the military domain. Research developments in military-based mission-critical systems have refined a lot as in autopilot, sensing true target behavior, battle damage conditions, acquiring and manipulating command control information. However, the application of pervasive systems in the military domain is still evolving. In this paper, an intelligent framework has been proposed for mission-critical systems to incorporate advanced heterogeneous communication protocols; service-oriented layered structure and context-aware information manipulation. The proposed framework addresses the limitation of "time-space" constraints in Mission-critical systems that have been improved significantly. This improvement is courtesy to enhancing situationaware tactical capabilities such as localization, decision significance, strategic span, strategic intentions, resource coordination and profiling concerning the situation. A comprehensive use case model has been presented for a typical battlefield scenario followed by a comparison of the proposed framework with existing techniques. It is evident from experiments and analyses that the proposed framework provides more effective and seamless interaction with contextual resources to improve tactical capabilities.
\end{abstract}

Keywords: Context-awareness, pervasive computing, middleware architecture, real-time systems, mission-critical systems, ubiquitous computing. 


\section{$1 \quad$ Introduction}

\subsection{Context Awareness in Pervasive Systems}

Ubiquitous computing is the method of enhancing computer usage by making many computers available throughout the physical environment while making them effectively invisible to the user. - Mark Weiser.

The ubiquitous or pervasive systems provide cutting edge technologies such as ambient intelligence, context-aware user interfaces, search engine abilities, and resource management through the integration of mobile computing and numerous data-collecting sensors [1]. Human emotional state, bio-physiological state and social interactions coupled with technology and tools provide potential meaningful information for situation awareness. Human perception requires two main processes for awareness (i) Perceptual Awareness (ii) Contextual awareness [2]. Applications customized to the context of the current working environment are motivated by the concept of enhancing interaction experience. Context-aware applications may have following models [3]:

1. Self-adaptive applications use context to transform behavior. Coupling of concerns/concepts in capturing the context adds to the complexity and scalability of pervasive systems.

2. Supervised adaptation depends upon directed logic and knowledge-based platform between applications and their context. Supervised based context-aware applications such as user navigation, identify user position at the sensory-level (context) while activity recognizing can be performed at the feature-level (application).

Storing multilayer information is resource consuming but also scalable. Layered system design is thus needed to incorporate major tasks of context-awareness in the pervasive systems [4]. Such design includes dynamic context detection of multiple resources, scalable and autonomous architecture with compound mechanisms for accessing the contextual information and decoupling of context.

When peripheral devices are attached to wireless devices, many critical mission actions can be performed such as multiple training scenarios, reporting individual and team skills, statistical and graphical analysis through repeated sessions. At the end of such critical missions, after-action-reviews (AARs) may be performed instead of waiting to make notes at the command or centralized arena [5]. Thus, generally speaking, pervasive systems are merged with time bond critical systems, they support:

1. Presentation of information and services to various stakeholders (user/operator, commander, controller)

2. Categorization of situational information i.e. location, vision, logistic support, political scenarios, etc.

3. Items for context illustration like maps, electronic markers, a global positioning system (G.P.S), body sensors, etc.

4. Organization of context attributes based on the schema for device discovery, knowledge base and transparency in user interaction. 
5. Impose integrity on resources and action disclosure information i.e. privacy.

6. Provide access control to multiple resources.

Current pervasive critical systems, start from room temperature sensing; security systems with sound and motion detectors; safety sensors in assembly/power plants and enhance to autopilot in passenger / military aircraft sensing flight parameters through various sensors.

\subsection{Design constraints of Context-Aware Pervasive Paradigm}

Humans have a great ability for perceiving, forecast, and understanding behavioral motivation. A collaboration of the context-aware computer with the situation-aware users would facilitate in developing a well-defined functional system. The monitoring of the situation-aware system includes obtaining data, organizing the acquired data based on a specific domain and inferring the resultant knowledge [6]. Major design concerns for context-awareness in MMC systems have been discussed in [7]

1. Choosing easy and pervasive interactions.

2. Designing aspects and materials should be specific to the capabilities of pervasive systems.

3. A team ware to have a single interactive display for multiple devices/resources.

4. Predictable input devices for a comprehensive interactive experience.

5. Applications to facilitate robust team interaction for collaborated design.

\subsection{Context-aware Mission Critical systems}

The capacity to access context-aware graphics, audio, textual data, and simulations in provides the possibilities for MMC (Man Mission Control) pervasive systems to carry out real-time events with augmented reality applications [8]. In MMC pervasive systems collaborate with virtual and real-world concurrently. As a soldier is contributing to the situation-aware era of pervasive computing, the data/information is changing the scenarios, based on situation relevant information provided by the technology-enabled tools.

The MMC paradigm is accomplished by applications such as face recognition, fingerprint authentication, motion sensors, physiological indicators (e.g., heartbeat rate, glucose levels), etc. To support MMC environment space; site maps, notes and tags would allow users to leave traces in the physical environment. A map has notes pasted in the form of chip /tag sensors and data is processed for contextual retrieval. Devices retrieve information from notes/tags to update map positions using technologies such as RFID, ZigBee, etc. Energy alertness, robust (information/situation) context acquisition and finding equilibrium between situational (context) sensing and power utilization to process data are research areas in the development of context-aware MMC-pervasive systems [9]. Security and reliance are also prevalent research domains of MMC- pervasive systems [9].

According to the perspective of "field user", the contextual information in the manmission control system of the battlefield has three broader categories: 
1. Type 'A': The soldier (field operator) has gadgets to provide latitude and longitude of positions to have actual terrain at command center (MMC system), visual range device to calculate accurately the target (interest areas), weather sensor, speedometer, team formation, medical fitness actuators, logistics reckon and route, invisibility support, and possible decision acquisitions. The snapshot of the field command software program depicts this type 'A' contextual information in Figure 1.

2. Type 'B': The command in the field (field commander) must-have situation awareness and transparency in decision making through group situation analytics, team locations, communication strength, and the field scenario recommendations by the MMC system. The field command software program depicts this type 'B' in Figure 2.

3. Type 'C': MMC system commands at CCI (Command and Control center) with an overall view of the tactics, current situation positioning of teams through satellite communications facilities, crisis history contexts for efficient paradigm analysis, geopolitical situation context with feedback (as shown in Figure 3).

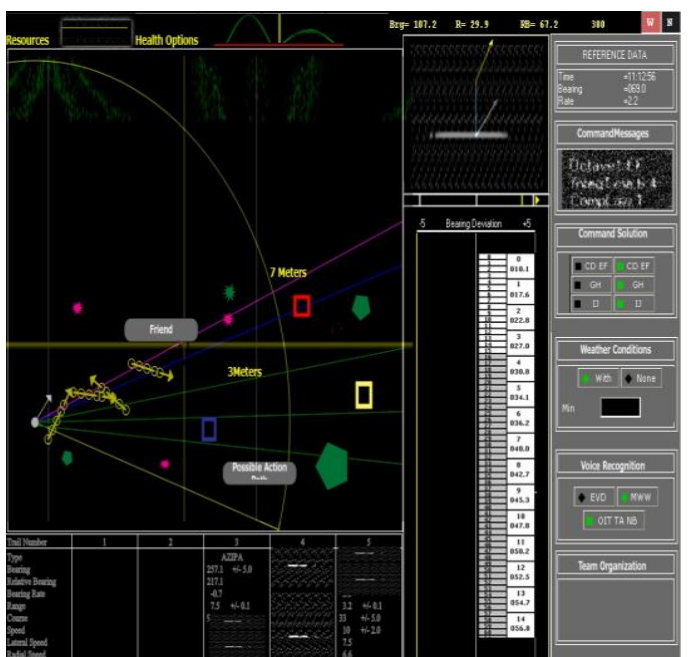

Fig. 1. Contextual information Type A - Field Operator Board

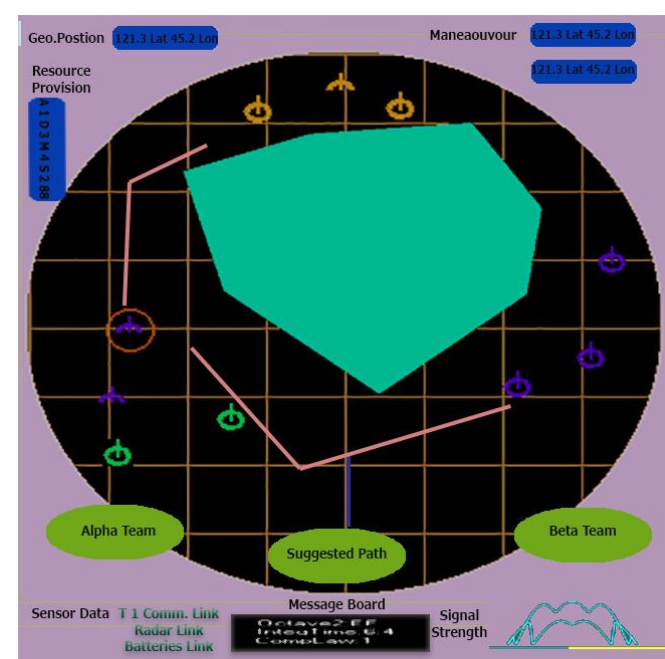

Fig. 2. Contextual information Type B - Field Commander Board 


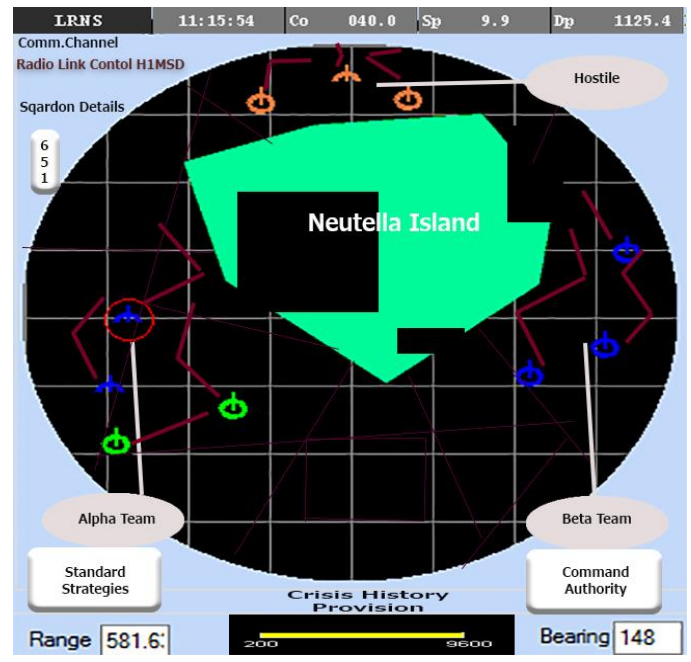

Fig. 3. Contextual information Type B - Command Authority Board

\section{4. Motivation}

Conventional Mission Critical frameworks lack facilities to adapt tactical situations and suggest confident information to enhance situation awareness for a timely response $[10,11]$. Most of the frameworks for mission-critical systems are based on the request/response paradigm such as High-Level Architecture [12] and Distributed Interactive Simulation [13]. Association or withdrawal of multiple resources is cumbersome due to the tight coupling of middleware services and communication standards. Moreover, current mission-critical frameworks lack situational awareness and transparency at all three levels of critical real-time environments namely individual, team, and command level [14].

The Context-aware MMC framework is proposed to provide context-aware of collaboration between devices in and across different environment spaces. Context-aware MMC is intelligent, a situation-aware service-oriented framework to support a wide range of mission-critical scenarios.

The rest of this paper is organized as follows: A brief background and related work are presented in section 2 . In section 3, the proposed framework for context-aware MMC IoT has been detailed. Section 4 presents a case study to illustrate the framework flow along with a simulation process, results and analysis. In section 5, a conclusion is provided with potential future work.

\section{Background and Related Work}

In recent years, advancements in information and communication technologies have brought an enormous change in human lives [15]. Regardless of challenges in manipulating context awareness with existing platforms and systems; pervasive computing has 
the ability to support especially in mission-critical domains to enhance the behavior of applications [16]. As real actions of real-time mission-critical systems are expensive, simulated training provide circumstances for active and decisive learning. Multiple training scenarios of such systems open up prospects for concurrent learning on multiple levels [17]; trainee/individuals may experience contextual information through the risks, benefits, expenses, results, and recompenses of alternate strategies that are instigated through the contextual information collected by sensors in the environment of mission-critical systems [18].

The emerging middleware software technologies and tools enable the distribution of real-time mission-critical aspects, making them tangible and affordable. Such frameworks help to simplify the development and optimization of different systems. Some mission-critical middleware is discussed in the following:

Adaptive and reflective middleware systems (ARMS) [12] provides a framework to simplify the development, optimization, and integration of middleware in real-time systems.

Distributed object computing (DOC) middleware [10] is composed of self-governing software objects, distributed throughout a wide range of networks to support a variety of middleware-based services.

The ACE ORB (TAO) [19] addresses the policies and mechanisms that span network adapters, operating systems, communication protocols, and Object Request Broker (ORB) middleware to meet the requirements of high-performance real-time applications.

The High-Level Architecture (HLA) [20] provides interface specifications, and object model templates as minimum essential tools for interoperability of appropriate tactical simulations.

Data Distribution Service (DDS) [13] is designed for enabling real-time data distribution. It is also, termed as Control Area Networks (CAN) for meeting real-time, distributed and parallel processing requirements.

Agent-based Context Management Framework (A-CMF) [21] is a context-aware management framework based on intelligent software agents.

A comparison with the existing distributed mission-critical framework approaches is summarized in Table - 1 .

Table 1: Comparison with other frameworks

\begin{tabular}{|l|l|l|l|}
\hline Frameworks & Heterogeneity & $\begin{array}{l}\text { Service-ori- } \\
\text { ented layer }\end{array}$ & $\begin{array}{l}\text { Context-aware } \\
\text { methodology }\end{array}$ \\
\hline $\begin{array}{l}\text { Context-aware } \\
\text { MMC }\end{array}$ & $\checkmark$ & $\checkmark$ & $\checkmark$ \\
\hline ARMS [12] & $\checkmark$ & $x$ & $x$ \\
\hline DOC [10] & $\checkmark$ & $\checkmark$ & $x$ \\
\hline TAO[19] & $\checkmark$ & $x$ & $x$ \\
\hline HLA[20] & $\checkmark$ & $\checkmark$ & $x$ \\
\hline DDS[13] & $\checkmark$ & $x$ & $x$ \\
\hline A-CMF [21] & $x$ & $x$ & $\checkmark$ \\
\hline
\end{tabular}


In recent years, context-aware computing has shown critical significance in pervasive computing [22] and plays an important role in the Mission Critical paradigms as well. Individuals and teams in mission-critical systems require situational awareness and transparency in multiple ways due to frequent movement in diverse work settings and engagement in parallel work activities. It results in various mutual problem-solving situations [23]. Similarly, Human-computer Interactions in mission-critical systems are fulfilled by considering a variety of electronic documents, schemas, electronic charts (ENC), touch boards, etc. All these documents are modified and prepared according to the particular work settings [24]. The situation awareness and transparency in such systems are realized by interacting/communicating diverse sensors and actuators used in the environment space pervasively. The integration of such contextual and situationaware resources in mission-critical systems can facilitate quick analysis and decision making.

\section{Proposed Framework - Context-Aware Man Mission Control (MMC)}

The use of multiple service layers forms the basis for context information at discrete layers requiring the execution and management of components independently [4]. Information in the form of messages also is instantiated using the triggered approach. This information is processed through subsequent layers in a service-oriented framework for related contexts. This results in specializing the context triggered and producing contextual semantics for an ongoing scenario/tactical environment. These activities ensure robustness, transparency and reduce ambiguity among subsequent layers. Activities and circumstances in a critical environment (scenario) do not exist in isolation, so the requirement of structuring the actions or contextual information (from general to specific) is also necessary for achieving contextual objectives.

Consider a list of contextual actions in environment defined by $\mathrm{A}=\left\{\mathrm{a}_{1}=\mathrm{aim}, \mathrm{a}_{2}=\right.$ position, ..., $\left.a_{n}\right\}$, where " $a_{n}$ " are the actions performed within a particular scenario $S_{c}$. The scenario $S_{c}$ comprising of multiple situations $D$ can be defined as $S_{c}=\sum t_{i=1} D_{i}$ and $\mathrm{D}_{\mathrm{i}}=\sum \mathrm{j}_{\mathrm{k}=1} \sum_{\mathrm{l}=\mathrm{k}}^{\mathrm{n}}\left(\mathrm{A}_{\mathrm{k}_{\mathrm{o}}} \mathrm{C}_{\mathrm{l}}\right)$, where $\mathrm{C}$ is the condition or constraints on contextual information (actions) of particular situation $\mathrm{D}_{\mathrm{i}}$. Constraints can be categorized as pre-action constrains represented by $\mathrm{E}$ or post-action constraints represented as $\mathrm{F}$, and mathematically $\mathrm{C}=\tau_{\mathrm{k}} \sum_{\mathrm{m}=1}^{\mathrm{n}}(\mathrm{E} \cap \mathrm{F})$ indicating the constraints interacting together within the situation $D_{i}$ to execute the scenario $S_{c}$. Within a particular scenario $S_{c}, F$ influences the generation of the contextual messages while $\mathrm{F}$ effects the contextual actions. i.e $\mathrm{E}(\mathrm{a}) \neq$ $\emptyset$ and $\mathrm{F}(\mathrm{a}) \neq \varnothing$. The contextual objective, $\mathrm{O}=\left\{\mathrm{o}_{1}=\right.$ weapon detection, $\mathrm{o}_{2}=$ localization tool, $\left.\ldots, \mathrm{O}_{\mathrm{n}}\right\}$ using context-aware MMC framework can be eased through contextual action A having contextual constraint vector $C$ affecting the situation $S_{c}$.

$\mathrm{O}\left(\mathrm{b} \in \mathrm{S}_{\mathrm{c}}\right)=\operatorname{argmax}\left[\sum_{\mathrm{d} \in \mathrm{D}} \sum_{\mathrm{a} \in \mathrm{A}}(\mathrm{d} . \mathrm{a})+\sum_{\mathrm{a} \in \mathrm{A}}\left(\prod_{\mathrm{i}=1}^{\mathrm{n}_{1}} \mathrm{P}\left(\mathrm{b}_{\mathrm{i}} \mid \mathrm{a}\right)+1 / \alpha \beta \prod_{\mathrm{j}=1}^{\mathrm{n}_{\mathrm{j}}}\left(\mathrm{C}_{\mathrm{j}} \mid \mathrm{b}, \mathrm{a}\right)\right)\right]$ 
Context aware MMC can be described in terms of contextual aspects with respect to resource context and application context.

\subsection{Resource Context View:}

A set of multiple and diverse services/devices interact with MMC framework in a heterogeneous environment [4]. Context-aware MMC supports heterogeneous communication protocols ranging from wide-area networks to personal/body networks. The layered structure of context-aware MMC ensures scalability of heterogeneous devices in the time bond critical environment through the Device Specific layer. This layer helps in keeping the history of resources contributing to contextual information through the Dynamic Resource Identifier. Resources once identified are then certified through Resource Registration for context-aware MMC framework. Pieces of evidence and records of certified contextual information concerning resources, machines, users or collaborated services are maintained using Resource Recovery. Finally, the resource information is forwarded to the context notification support layer. The context notification support layer asynchronously notifies the agents about their contextual changes. The flow chart of the resource context process is shown in Figure 4. 


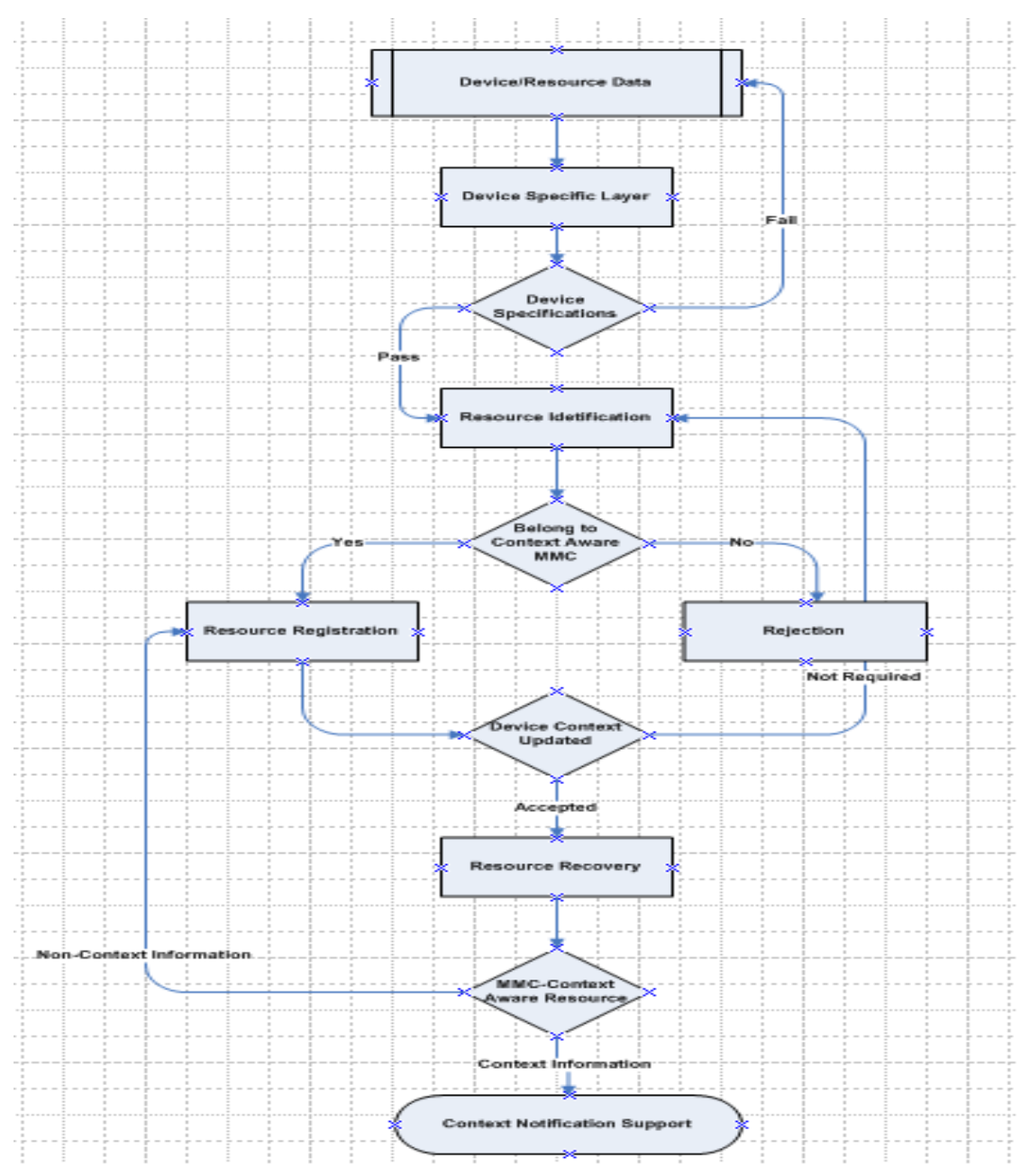

Fig. 4. Resource Context Broker View of Context-aware MMC framework

\subsection{Application Context View:}

Module termed as Agents are abstractions to represent sensors and services in Contextaware MMC. Agents can either be monitors or actuators. Those are responsible for acquiring data from multiple resources in environment space and distributing it to further levels in context-aware MMC framework (monitors). When situational action interacts, agents manipulate the contextual information and forward refined information to further levels (actuators). Context-aware MMC put the refined information on the notice-board (NB) i.e. field operator, field commander or command authority boards. The flowchart in Figure 5 depicts the application context process. 


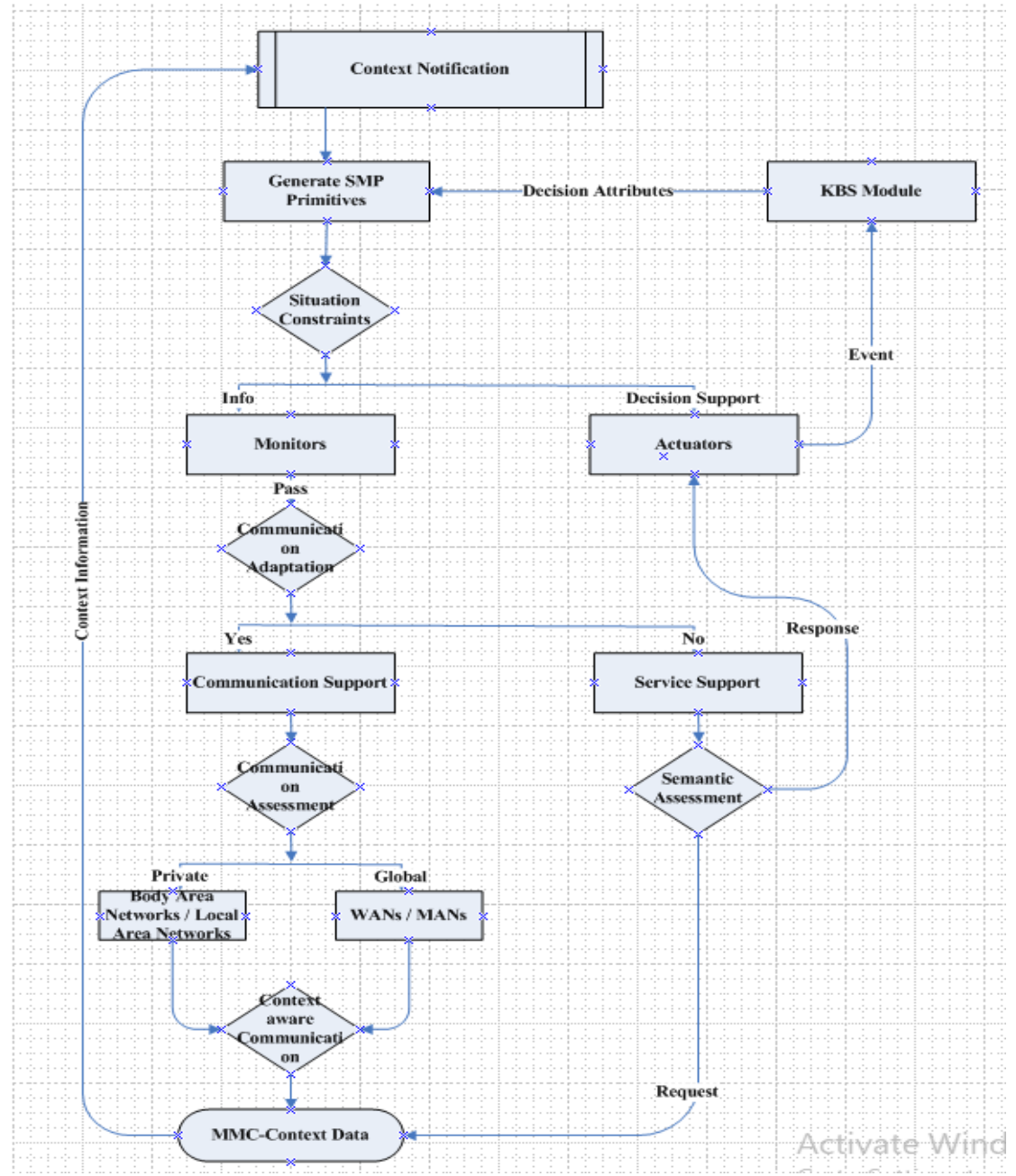

Fig. 5. Application Context Broker View of Context-aware MMC framework

The information manipulation methodology in MMC framework focuses on obtaining data, organizing the relevant data and inferring domain-specific knowledge. Information manipulation in MMC uses a supervised adaptation model [25].

Context-aware MMC framework provides internal self-describing services to maintain the access information of distributed resources.

Device Support: This segment working at the device-specific layer provides support for routing and forwarding information among physical devices e.g. sensors, gadgets etc. which are interacting in a real time-critical environment.

Resource Support: This segment also working at a device-specific layer that provides resource recovery and registration along with a dynamic identifier module to manipulate information form machine, user or services acting as resources for context-aware MMC. 
Communication Support: This supports the broadcast and reception of context-aware information datagram messages exchanged between various layers. Context-aware MMC enabled devices do not have the same set of communication technologies. A sensor node might be equipped with ZigBee or WiMax while resources/human might be equipped with pervasive support devices like blood pressure levels, pulse rates, heartbeat rates, etc connected through energy-efficient technologies. So to communicate heterogeneous devices, it is necessary to have a base layer to act as a gateway as shown in Figure 6.

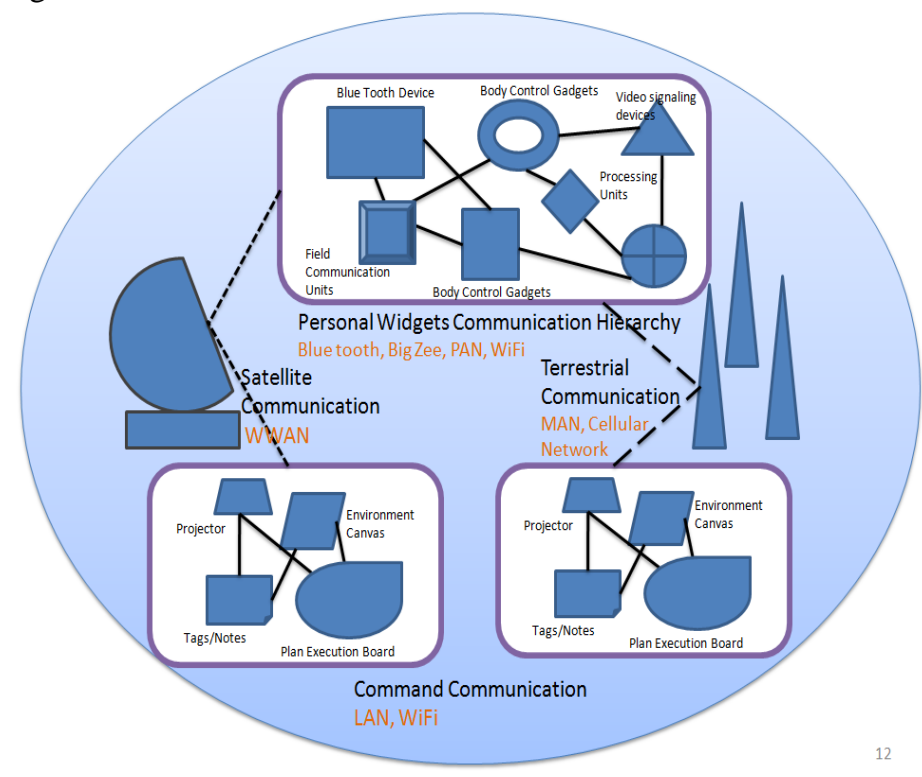

Fig. 6. Communication Technologies in MMC

Service Support: It provides a request-response semantic for the context-aware MMC framework. Context-aware MMC framework has helper modules in the service support layer. Query mechanism, I/O for communication, resource handling and dynamic checkpoint services used for searching data, communicating and fault tolerance respectively, are using semantics provided by service support as shown in Figure 7.

Context Notification Support: It allows context-aware MMC framework to store, analyze and process contextual information. Context information can be retrieved by monitors/actuators using a simple message protocol (SMP) [26]. Simple Message Protocol (SMP), is a text-based protocol to access context data and provide support for heterogeneity. This is a simple protocol used to create a robust layered context-aware MMC infrastructure, where distributed context notification services can collaborate. Ambiguity removal and context accretions are performed in this layer using knowledge base support (KBS).

Context Environment (Mission Critical Environment): Environment of missioncritical space is responsible for providing context-aware scenarios in context-aware 
MMC framework. Multiple environments provide diverse situational, physical and social awareness of context-aware scenarios. This blend of real and virtual context-aware features and attributes enables efficient response to critical situations and experiences as shown in Figure 8.

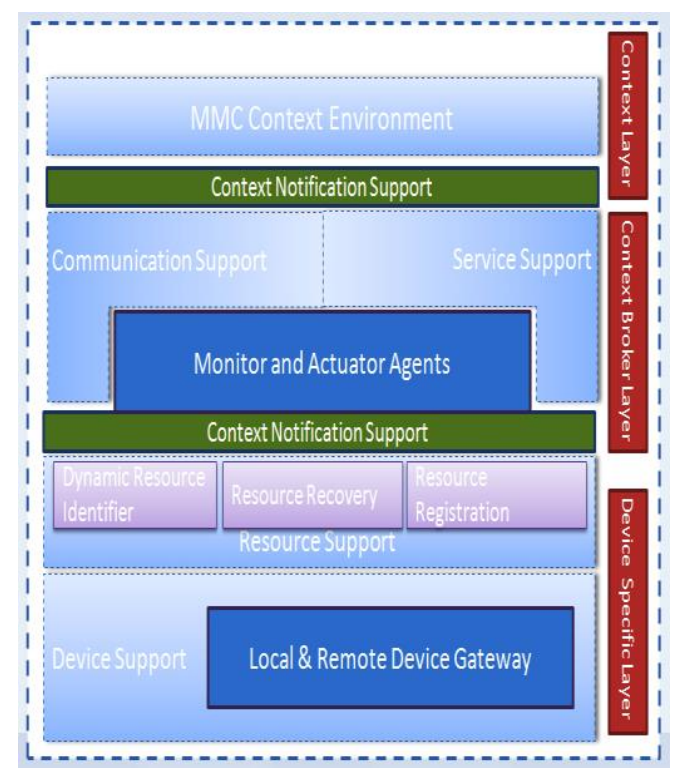

Fig. 7. Layered Architecture of Context-aware MMC framework

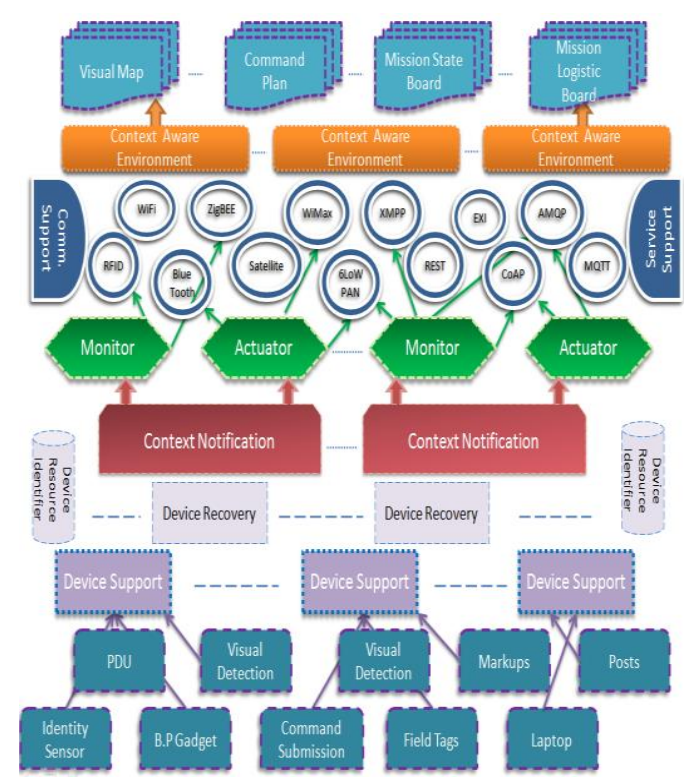

Fig. 8. Context-aware MMC framework Interactions

\section{$4 \quad$ Analysis and Evaluation}

To analyze the behavior of the proposed framework, a use case model based on Unified Modeling Language (UML) 2.5 is presented in this section. For proof-of-concept, this proposed framework is applied to a typical battle-field scenario. Computational efficiency and tactical parameter interactions have been analyzed through simulations performed using a Context-aware MMC Emulator (simulator) on a distributed network of computers. The network comprised of 10 computers equipped with an Intel Core i7 processor operating at $3.4 \mathrm{GHz}$ and $8 \mathrm{~GB}$ of memory using GPU of $8 \mathrm{~GB}$. These are the optimal parameters decided after experiment scenarios.

\subsection{Use Case Analysis:}

The target localization phase, as shown in Figure 9, within the battlefield using contextaware MMC [27]. It depicts a battlefield scenario when a soldier in the field finds the enemy movement through binocular; the strategic information is shared among tactically spanned field commander and command center. At this point, the context-aware 
MMC framework contributes to evaluating available resource profiles which in this situation includes seamless calculation of several rounds, caliber, weapon selection, laser range finder etc. It also supports selecting the nearest and appropriate support team to establish communication with an exact position. This is very crucial for a soldier (actor), where a timely action is highly desirable. MMC Context environment further performs adjustment of gun seamlessly by calculating range and bearing of the intended target while keeping in view the terrain, weather condition, environment (jungle, mountain, river etc). All this information which includes resource profiling, target localization and tactical intention is shared with the tactically spanned command center. In this situation, the command center has a significant role in improving decision significance of tactical information thereof proposed by context-aware MMC to improve tactical actions and perform strategic movements within the battlefield by monitoring and controlling the tactically spanned field soldiers and weaponry assets (resource coordina-

tion) as shown in Figure 10.

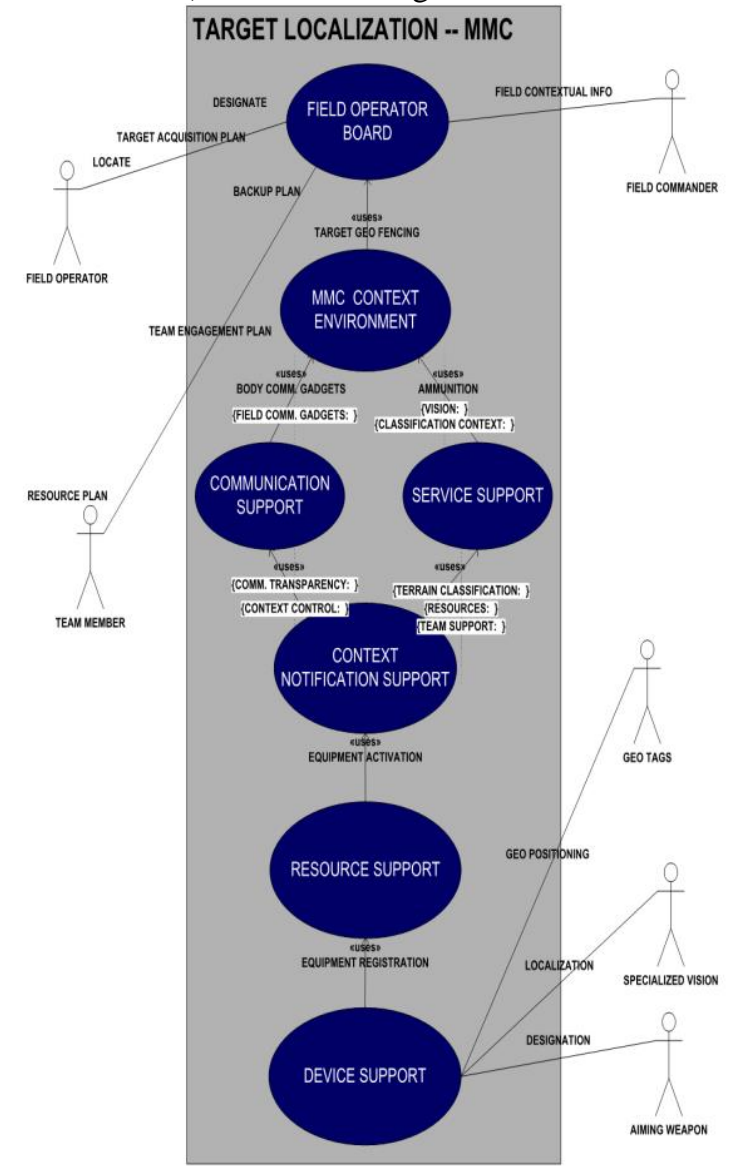

Fig. 9. Field Scenario using Context-aware MMC

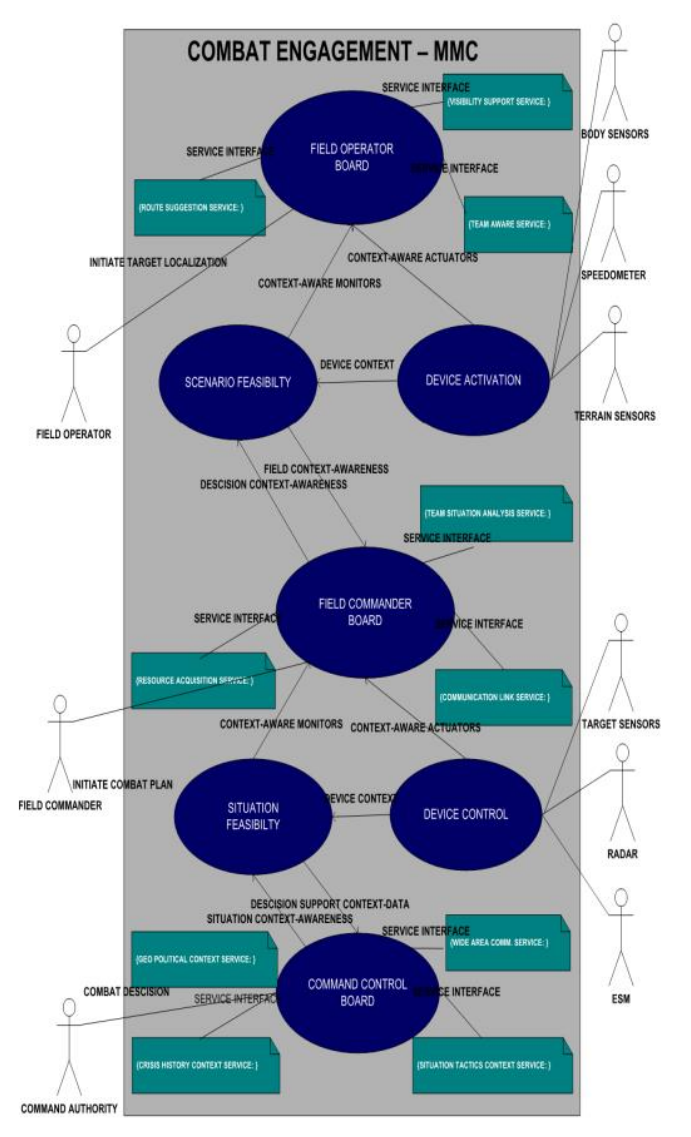

Fig. 10. Command Center Scenario using Context-aware MMC 
Similarly, the command center by using context-aware MMC for the given battlefield provides a proactive approach. This situation updates on a tactical intention to the field commander that the identified enemy movement is the movement of a neutral actor (Red Cross) for rendering life support to injured through geopolitical context suggestions. The field actors (soldiers) are provided with updated tactical intentions with higher decision significance about changed situations, as ordered by the field commander to remain to halt their positions.

\subsection{Experimental Analysis:}

Several parameters ranging from memory, I/O and network communication, distributed services till heterogeneous contextual resources/sensors and pattern evaluation services are involved in context-aware MMC framework to amicably and transparently handle the tactical situation awareness. As in tactical situations, space-time is decisive, parameters in Table 2 are evaluated for a scenario in section 4.1.

It may be observed from Figure 11 that resource profiling plays a key role since the start of context-aware MMC simulation in the current scenario and ceases with time as the situation develops. Tactical span events also enhance situation awareness initially by establishing (soldier- field commander) collaboration and then further to (filed commander - command center) levels. Decision significance events contribute to context awareness (after some time - $20 \mathrm{sec}$ ) as the situation evolves and context-aware MMC framework began evaluating the current situation. The tactical intention graph suggests a decrease in corresponding events as the system concludes to a possible solution for the tactical situation in section 4.1.

Table 2. Test-bench parameters for Context-aware MMC Emulator

\begin{tabular}{|l|l|}
\hline $\begin{array}{c}\text { Context-aware MMC Tacti- } \\
\text { cal Parameters }\end{array}$ \\
\hline Decision Significance & DS \\
\hline Resource Profiling & RP \\
\hline Tactical span & TS \\
\hline Tactical Intentions & TI \\
\hline
\end{tabular}

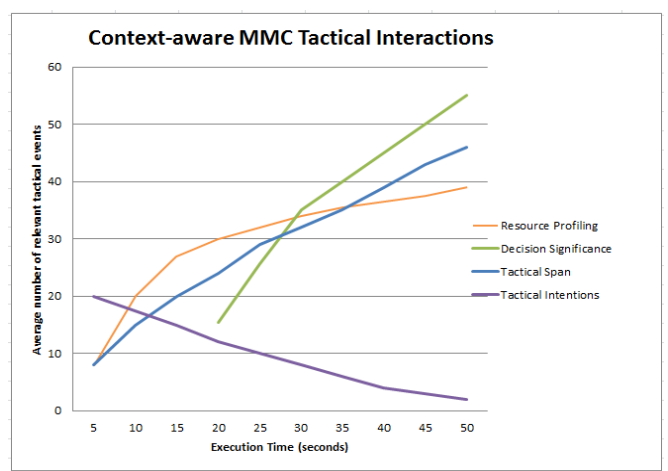

Fig. 11. Interactions of tactical capabilities in Context-aware MMC Emulator 
The increase of context-aware units (events) qualified to take part in the current scenario increases as the system interacts, thus making context-aware units a reliable factor to analyze the performance of situation awareness. Analyzing contextual aware units in context-aware MMC Emulator gives a glimpse of our proposed framework efficiency. It can be observed in Figure 12 that contextual performance of the proposed paradigm increases (almost exponentially) with time due to frequent interaction of contextual units in the discussed scenario, thus resulting in better context-awareness.

Another perspective to evaluate the performance of our proposed context-aware MMC framework is several periodic training sessions conducted using the emulator.

The current situation is optimized through repeated sessions as shown in Figure 13. Training time is observed to decrease in learning the contexts. The contextual information (red line) is continually updated, resulting in the better parameterization of contextual units. After about 2 days of training with the scenario discussed in section 4.1, the experiment shows that the system achieves situational robustness and interactivity of contextual resources.

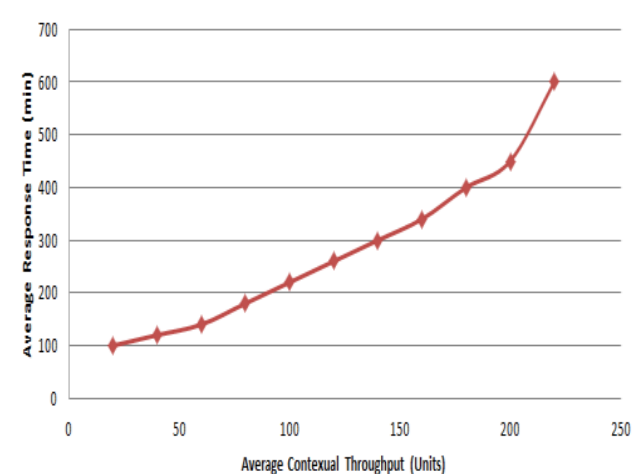

Fig. 12. Analysis of Contextual Response

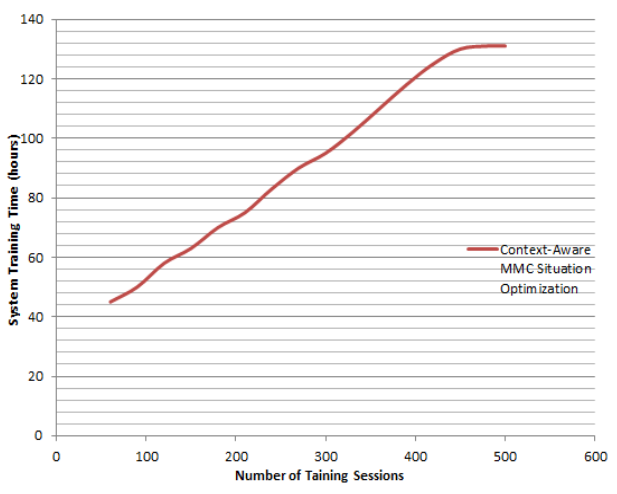

Fig. 13. Contextual Situation Optimization

\section{Conclusion and Future Work}

The context-aware MMC paradigm supports heterogeneous contextual data processing to enhance tactical capabilities to mitigate time-space constraints. It has been demonstrated for a typical battle-field scenario in a use-case model followed by evaluation. A comparison with existing paradigms has also been performed that empirically asserts the proposed technique as more efficient and effective.

In the future, ever-growing diversity and reduced complexity demand for other mission-critical systems would be considered. It requires the teams to process information more quickly and ubiquitously. Quest for searching, merging and transmitting diverse 
information about a critical situation, statistics about teams and technical contextual data is a challenge. Endeavor to implement this model in similar critical domains like power plant operations, and disaster management teams may provide insights into context-aware and context un-aware systems.

\section{References}

[1] N. Bricon-Souf and C. R. Newman, "Context awareness in health care: A review," international journal of medical informatics, vol. 76, pp. 2-12, 2007.

[2] M. Gross, "Context-Aware Computing: From Neuroscience to Mobile Devices," 2015.

[3] M. Dalmau, P. Roose, and S. Laplace, "Context aware adaptable applicationsa global approach," arXiv preprint arXiv:0909.2090, 2009.

[4] E. Matsas and G.-C. Vosniakos, "Design of a virtual reality training system for human-robot collaboration in manufacturing tasks," International Journal on Interactive Design and Manufacturing (IIIDeM), vol. 11, pp. 139-153, 2017/05/01 2017.

[5] R. Parasuraman, M. Barnes, K. Cosenzo, and S. Mulgund, "Adaptive automation for human-robot teaming in future command and control systems," Army Research Lab Aberdeen Proving Ground Md Human Research And Engineering Directorate, 2007.

[6] N. Koshizuka and K. Sakamura, "Ubiquitous ID: standards for ubiquitous computing and the Internet of Things," IEEE Pervasive Computing, vol. 9, pp. 98-101, 2010.

[7] J. Grubert, T. Langlotz, S. Zollmann, and H. Regenbrecht, Towards Pervasive Augmented Reality: Context-Awareness in Augmented Reality vol. 23, 2016.

[8] A. Zanella, N. Bui, A. Castellani, L. Vangelista, and M. Zorzi, "Internet of things for smart cities," IEEE Internet of Things journal, vol. 1, pp. 22-32, 2014.

[9] J. Gubbi, R. Buyya, S. Marusic, and M. Palaniswami, "Internet of Things (IoT): A vision, architectural elements, and future directions," Future generation computer systems, vol. 29, pp. 1645-1660, 2013.

[10] D. C. Schmidt, "Middleware for real-time and embedded systems," Communications of the ACM, vol. 45, pp. 43-48, 2002.

[11] C. D. Gill, F. Kuhns, D. L. Levine, et al., "Applying adaptive real-time middleware to address grand challenges of COTS-based mission-critical real-time systems," IEEE International Workshop on Real-Time Mission-Critical Systems: Grand Challenge Problems, 1999.

[12] M. Ficco, G. Avolio, F. Palmieri, and A. Castiglione, "An HLA-based framework for simulation of large-scale critical systems," Concurrency and Computation: Practice and Experience, vol. 28, pp. 400-419, 2016.

[13] T. Guesmi, R. Rekik, S. Hasnaoui, and H. Rezig, Design and Performance of DDS-based Middleware for Real Time Control Systems, 2018.

[14] F. Ciccozzi, I. Crnkovic, D. Di Ruscio, "Model-Driven Engineering for MissionCritical loT Systems," IEEE Software, vol. 34, pp. 46-53, 2017. 
[15] A. K. Dey, G. D. Abowd, and D. Salber, "A conceptual framework and a toolkit for supporting the rapid prototyping of context-aware applications," Humancomputer interaction, vol. 16, pp. 97-166, 2001.

[16] C. Stergiou, K. Psannis, B.-G. Kim, and B. B. Gupta, Secure integration of IoT and Cloud Computing vol. 78, 2016.

[17] C. Sellberg, "From briefing, through scenario, to debriefing: the maritime instructor's work during simulator-based training," Cognition, Technology \& Work, vol. 20, pp. 49-62, 2018/02/01 2018.

[18] P. Bloom and Q. Chung, "Lessons learned from developing a mission-critical expert system with multiple experts through rapid prototyping," Expert Systems with Applications, vol. 20, pp. 217-227, 2001.

[19] D. C. Schmidt, D. L. Levine, and S. Mungee, "The Design of the TAO Real-Time Object Request Broker," Computer Communications, 1999.

[20] J. S. Dahmann, R. M. Fujimoto, and R. M. Weatherly, "The department of defense high level architecture," $29^{\text {th }}$ conference on Winter simulation, pp. 142-149, 1997

[21] I. Anghel, T. Cioara, I. Salomie, and M. Dinsoreanu, "An Agent-based Context Awareness Management Framework," $8^{\text {th }}$ International Conference RoEduNet, pp. 107-113, 2009

[22] K. Henricksen, J. Indulska, and A. Rakotonirainy, "Modeling context information in pervasive computing systems," Pervasive Computing, pp. 79-117, 2002.

[23] D. H. Sonnenwald and L. G. Pierce, "Information behavior in dynamic group work contexts: interwoven situational awareness, dense social networks and contested collaboration in command and control," Information Processing \& Management, vol. 36, pp. 461-479, 2000.

[24] J. S. Tucker, "Mobile learning approaches for US Army training," Army Research Inst For The Behavioral And Social Sciences Fort Benning 2010.

[25] R. Calinescu, M. Autili, J. Cámara, A. Di Marco, S. Gerasimou, P. Inverardi, et al., "Synthesis and Verification of Self-aware Computing Systems," in Self-Aware Computing Systems, S. Kounev, J. O. Kephart, A. Milenkoski, and X. Zhu, Eds., ed Cham: Springer International Publishing, pp. 337-373, 2017

[26]. Hawley, John Alfred (June 1975). "MUNIX, A Multiprocessing Version Of UNIX" (PDF). core.ac.uk. Retrieved 11 November 2018.

[27] J. Fradley, R. Preece, Assessment of the Impact of MMC-VSC Intrinsic, Energy on Power System Stability, The Journal of Engineering, 2019 